\title{
Epithelial innate immunity mediates tubular cell senescence after kidney injury
}

\author{
Heng Jin, , ${ }^{1,2}$ Yan Zhang, ${ }^{1,2}$ Qiong Ding, ${ }^{1}$ Shan Shan Wang, ${ }^{3}$ Prerna Rastogi, ${ }^{4}$ Dao-Fu Dai, ${ }^{4}$ \\ Dongmei Lu, ${ }^{5}$ Madison Purvis, ${ }^{1}$ Chao Cao, ${ }^{1,2}$ Angela Wang, ${ }^{1}$ Dingxiao Liu, ${ }^{1}$ Chongyu Ren, ${ }^{5}$ \\ Sarah Elhadi, ${ }^{5}$ Ming-Chang Hu, ${ }^{5}$ Yanfen Chai, ${ }^{2}$ Diana Zepeda-Orozco, ${ }^{6}$ Judith Campisi, \\ and Massimo Attanasio ${ }^{1}$ \\ 'Department of Internal Medicine, University of lowa, lowa City, lowa, USA. ${ }^{2}$ Department of Emergency Medicine, \\ Tianjin Medical University General Hospital, Tianjin, China. ${ }^{3}$ Zhongshan Hospital, Fudan University, Shanghai, China. \\ ${ }^{4}$ Department of Pathology, University of lowa, lowa City, lowa, USA. ${ }^{5}$ Department of Internal Medicine, University of Texas \\ Southwestern Medical Center, Dallas, Texas, USA. ${ }^{6}$ Department of Pediatrics, University of lowa, lowa City, lowa, USA. \\ ${ }^{7}$ The Buck Institute for Research on Aging, Novato, California, USA.
}

Acute kidney injury (AKI) is a common clinical condition of growing incidence. Patients who suffer severe AKI have a higher risk of developing interstitial fibrosis, chronic kidney disease, and endstage renal disease later in life. Cellular senescence is a persistent cell cycle arrest and altered gene expression pattern evoked by multiple stressors. The number of senescent cells increases with age and even in small numbers these cells can induce chronic inflammation and fibrosis; indeed, in multiple organs including kidneys, the accumulation of such cells is a hallmark of aging. We hypothesized that cellular senescence might be induced in the kidney after injury and that this might contribute to progressive organ fibrosis. Testing this hypothesis, we found that tubular epithelial cells (TECs) in mice senesce within a few days of kidney injury and that this response is mediated by epithelial Toll-like and interleukin 1 receptors (TLR/IL-1R) of the innate immune system. Epithelial cell-specific inhibition of innate immune signaling in mice by knockout of myeloid differentiation $\mathbf{8 8}$ (Myd88) reduced fibrosis as well as damage to kidney tubules, and also prevented the accumulation of senescent TECs. Importantly, although inactivation of Myd88 after injury ameliorated fibrosis, it did not reduce damage to the tubules. Selectively induced apoptosis of senescent cells by two different approaches only partially reduced kidney fibrosis, without ameliorating damage to the tubules. Our data reveal a cell-autonomous role for epithelial innate immunity in controlling TEC senescence after kidney injury, and additionally suggest that early therapeutic intervention is required for effective reduction of long-term sequelae of AKI.

Authorship note: $\mathrm{HJ}$ and $\mathrm{YZ}$ contributed equally to this work.

Conflict of interest: The authors have declared that no conflict of interest exists.

License: Copyright 2019, American Society for Clinical Investigation.

Submitted: October 11, 2018 Accepted: December 11, 2018 Published: January 24, 2019

Reference information: JCI Insight. 2019;4(2):e125490. https://doi.org/10.1172/jci. insight.125490.

\section{Introduction}

Acute kidney injury (AKI) is a common clinical affliction and an increasing cause of mortality. Although AKI has been considered a self-limited condition, the existence of an association between recurrence, the severity of AKI episodes, and subsequent progression to chronic kidney disease (CKD) have been recently recognized (1-3). Tubule repair is a common event after kidney injury, but is frequently associated with interstitial inflammation and maladaptive processes that lead to fibrosis, the hallmark of all forms of kidney disease and a reliable predictor of progression to CKD (4). Recently, multiple studies identified multipotent mesenchymal stromal progenitor cells (pericytes) as the cell population that is responsible for collagen deposition after injury, in response to Hedgehog-mediated (Hh-mediated) proproliferative and profibrogenic signaling from the epithelium $(5,6)$. Kidney fibrosis has also been correlated with arrest of tubular epithelial cells (TECs) in the G2/M phase of the cell cycle (7-9), which suggests that the epithelium plays a primary role in the progression of kidney disease. However, the factors that contribute to the cell cycle arrest are not known. Cell cycle arrest is a universal marker of cellular senescence and is evoked by a variety of stressors, including oncogene activation, replicative stress, replicative exhaustion, telomere dysfunction, and oxidative stress ( 10 , 11). Senescent cells are resistant to apoptosis and their persistence in tissues, even in small numbers, can cause chronic inflammation and fibrosis, an aging-associated phenotype in multiple organs, including the kidneys $(12,13)$. Senescent cells communicate with the neighboring tissue by secreting a heterogeneous assortment of 
molecules. This results in the senescence-associated secretory phenotype (SASP), which is controlled by multiple pathways, among which NF- $\mathrm{BB}$ is prominent (14-16). SASP signaling is a double-edged sword because, although it can promote reparative programs in the short term, it also sustains proinflammatory processes and the further accumulation of senescent cells in the long term $(17,18)$.

Several studies have reported a correlation between the presence of senescent cells and kidney fibrosis both during the aging process and in the context of disease (19-21), but a systematic study of cellular senescence after AKI and its potential contribution to the progression of tubular damage and fibrosis is lacking (22). Here, we show that in mice TECs commonly become senescent after various types of kidney injury, and that this occurs surprisingly early after injury. We show that senescent TECs express higher levels of proinflammatory factors of the SASP as a result of cell-autonomous control by the TLR/IL-1R-mediated innate immune signaling pathway, and that senescent TECs are a source of the mesenchymal progenitor-activating ligands Sonic Hedgehog (Shh) and Indian Hedgehog (Ihh). Tubule-specific inhibition of TLR/IL-1R signaling by conditional inactivation of the Myd88 gene prior to senescence not only reduced the levels of epithelial cell-derived proinflammatory cytokines, interstitial infiltration, and fibrosis, but also decreased the accumulation of senescent cells and ameliorated tubular damage. Whereas inactivation soon after injury was equally effective in decreasing the number of senescent TECs, inflammation, and fibrosis, it did not protect from tubular damage. Similarly, eliminating $\mathrm{p} 16^{+}$senescent cells, but not senescent cells by FOXO4-DRI inhibitory peptide, which induces apoptosis of senescent cells by disrupting the interaction between FOXO4 and p53, reduced kidney fibrosis without reducing tubular damage. Our results indicate that TEC senescence is a common and early event after kidney injury, and that signaling by the TLR/ IL-1R pathway within the epithelium controls this phenomenon in a cell-autonomous fashion. Our findings also suggest that early intervention after injury is likely required to reduce organ damage after AKI. Furthermore, compared with published studies that focused on the role of the innate immunity signaling in pericytes, this study reveals what we believe is a novel function of the epithelial TLR/IL-R1 signaling in controlling the onset of TEC senescence in a cell-autonomous manner, and the proliferation and the cell fate of pericytes non-cell autonomously, consistent with the concept that the tubular epithelium triggers kidney disease following injury and also drives its progression.

\section{Results}

AKI induces cell senescence in TECs. To test whether cellular senescence is a common event after kidney injury, we looked for 2 established hallmarks of senescence: an increase in activity of the enzyme senescence-associated $\beta$-galactosidase (SA- $\beta-\mathrm{Gal}$ ), and a reduction in abundance of lamin B1 (LAMNB1) protein in the nuclear envelope (23). We used 3 mouse models of kidney injury: folic acid-induced (FA-induced) nephrotoxicity, ischemia/reperfusion injury (IRI), and cisplatin-induced (CP-induced) nephrotoxicity. We assessed senescence 28 days after the initial insult. In TECs of all 3 injury models, SA- $\beta$-Gal activity increased (Figure 1, A-F) and LAMNB1 levels decreased (Figure 1, G-L). These results suggest that TEC senescence is common to several forms of AKI.

Tubular cells undergo senescence early after kidney injury. To further characterize the onset of cellular senescence in tubular cells after AKI, we took advantage of the $p 16-3 M R$-transgenic mouse line (24). These mice had been generated by cloning the gene encoding the trimodality reporter (3MR) fusion protein, which contains functional domains of a synthetic Renilla luciferase (LUC), monomeric red fluorescent protein (mRFP), and truncated herpes simplex virus 1 (HSV-1) thymidine kinase (HSV-TK) (25), in frame with the promoter of the tumor suppressor gene $p 16^{\text {Ink4a }}$, the expression of which is activated in senescent cells. mRFP permits flow cytometry-based sorting of the expressing cells from tissues, and HSV-TK allows their killing by administration of ganciclovir (GCV), a nucleoside analog that has a high affinity for TK from HSV but a low affinity for its mammalian counterpart (cellular TK). HSV-TK converts GCV into a toxic DNA chain terminator that induces fragmentation of mitochondrial DNA in nondividing senescent cells and causes death by apoptosis (26). For the experiments described below, we chose not to use either the CP or IRI injury model because CP induces DNA damage and senescence and IRI-induced damage is not confined to tubular cells. We therefore focused on the FA model, using a single injection of FA that is toxic to rodent TECs because it causes intraluminal precipitation of folate crystals and oxidative stress (27-29). By confocal immunofluorescence microscopy, we consistently observed expression of mRFP, in tubular segments mostly at the cortico-medullary junction (Figure 2A and Supplemental Video 1; supplemental material available online with this article; https://doi.org/10.1172/jci.insight.125490DS1). This signal 
A

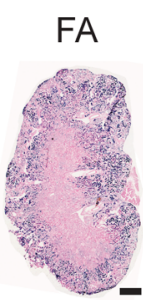

B
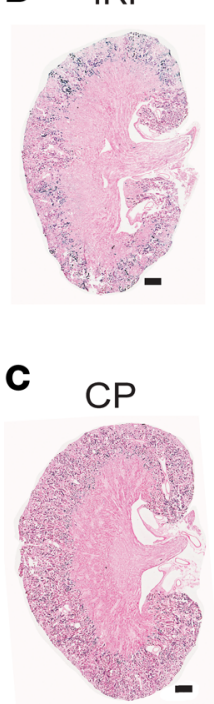

D

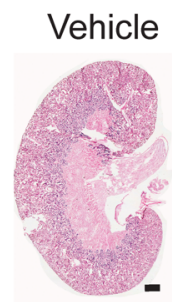

Sham

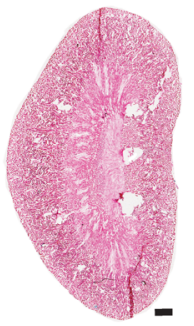

Vehicle

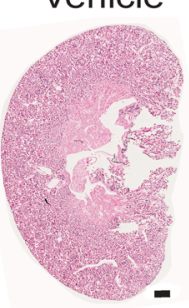

E

$\mathbf{F}$
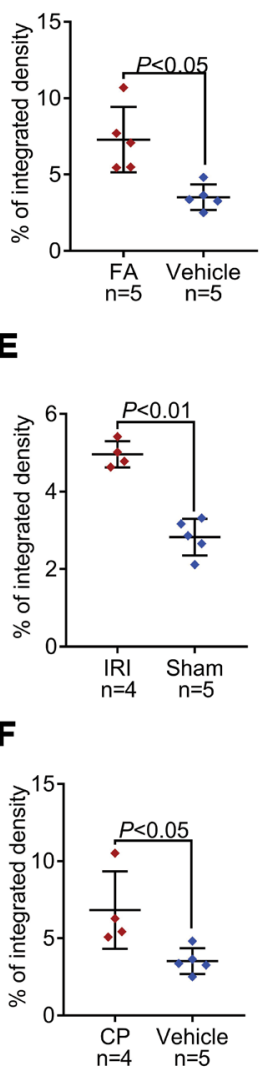

G
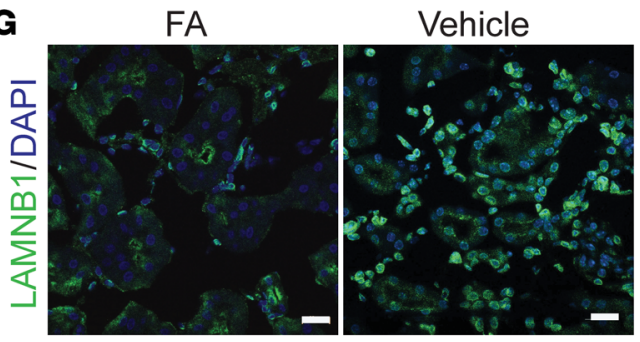

H
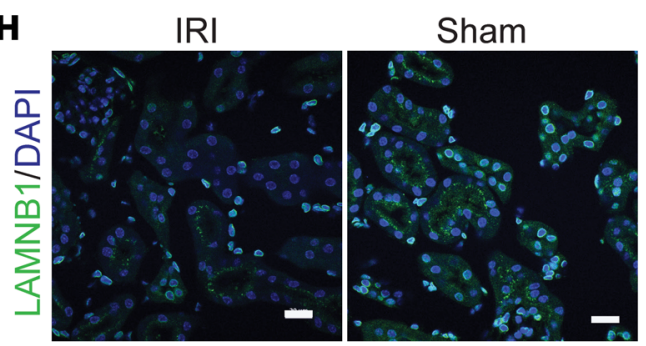

I

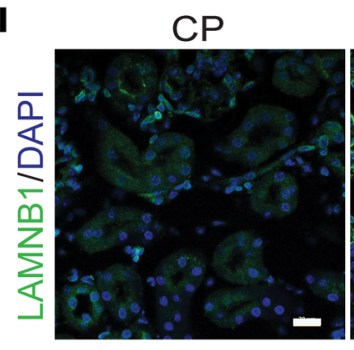

J

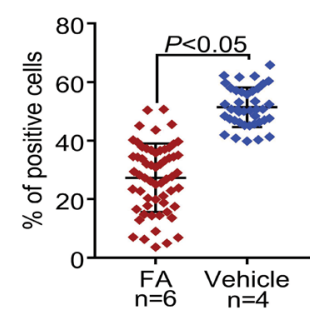

K

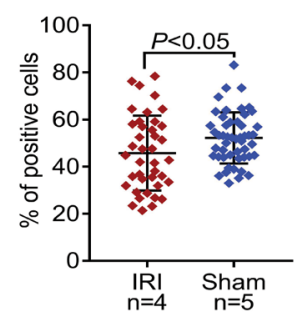

$\mathbf{L}$

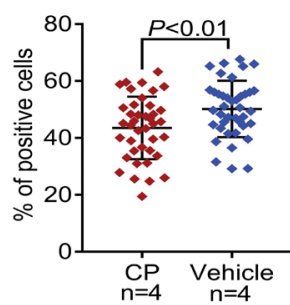

Figure 1. AKI induces cell senescence in kidney tubular cells. (A-C) SA- $\beta$-Gal staining of kidneys in 3 mouse models of AKI (FA, IRI, and CP) 28 days after injury, compared with controls and relative quantification (D-F). Scale bars: $500 \mu \mathrm{m}$. (G-I) Representative immunofluorescence confocal images of LAMNB1 28 days after FA, IRI, and CP, and relative digital image analysis qualification of LAMNB1-positive cells (J-L). Scale bars: $20 \mu \mathrm{m}$. Data are presented as mean \pm SD. $P$ values were calculated with 2-tailed Student's $t$ test. Ten images per mouse. The numbers of experimental mice are indicated in each panel.

frequently colocalized with the proximal-tubule marker Lotus tetranoglobus lectin (LTL, Figure 2B) but not with the collecting-duct marker Dolichos biflorus agglutinin (DBA, not shown). Surprisingly, quantification of mRFP fluorescence in explanted kidneys (Figure 2C) showed that, in the context of FA injury, levels of senescence were significantly higher than in vehicle-injected controls as early as 3 days after FA injury, and that the levels increased slowly at later time points (Figure 2D). The appearance of senescent cells in the tubules between day 2 and day 3 after injury was confirmed by staining for SA- $\beta$-Gal activity (Supplemental Figure 1), indicating that TEC senescence is an early event after injury.

Epithelial cell-specific deletion of Myd88 reduces expression of proinflammatory cytokines after FA injury. Senescent cells express many proinflammatory genes as part of the SASP and whose appearance and persistence are largely controlled by NF- $\mathrm{kB}(14-16,23)$. IL-1R is an important mediator of the paracrine effects of the SASP and activates NF-kB by recruiting the adapter protein Myd88 (30). Myd88-dependent activation of $\mathrm{NF}-\mathrm{KB}$ is also key to signaling downstream of the TLRs $(31,32)$. The roles of TLR signaling and Myd88 as mediators of inflammation have been extensively studied in several pathological contexts, including AKI, where this signaling axis controls the migration of pericytes, their conversion to myofibroblasts, and the secretion of proinflammatory cytokines from the latter (33-35). However, TLRs 1-4 are also constitutively expressed in both the proximal and distal tubules, in the thick ascending limb of the loop of Henle, and in the collecting ducts, suggesting that epithelial TLR signaling could also contribute to the development of inflammation and fibrosis after AKI (36). Because epithelial TLR2, TLR4, and Myd88 are upregulated in animal models of AKI $(36,37)$, we hypothesized that activation of TLR/IL-1R signaling in TECs can sustain the tubule-derived SASP after injury.

To test this hypothesis, we used the FA model of injury in mice with kidney tubule-specific deletion of $M y d 88$ ( $\left.{ }^{\mathrm{Ksp}-\mathrm{Cre}} \mathrm{Myd} 88^{n / f f}\right)$, in which expression of the Cre transgene is driven by the promoter of 
A

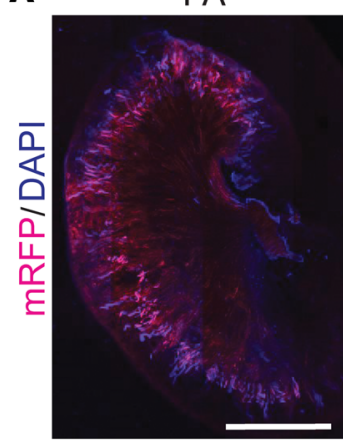

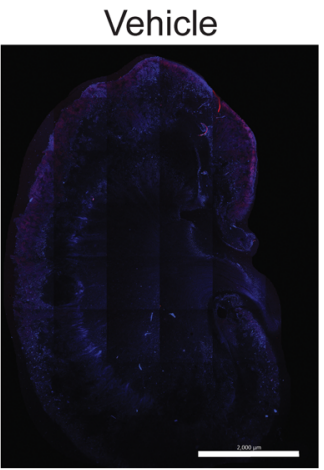

B

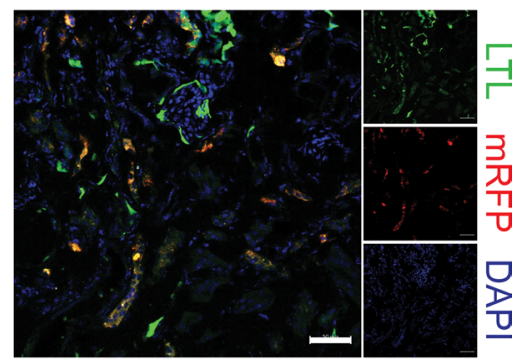

C

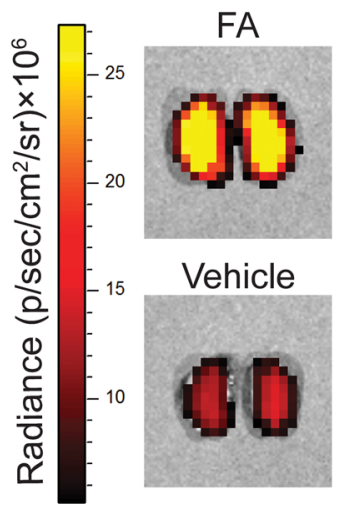

D

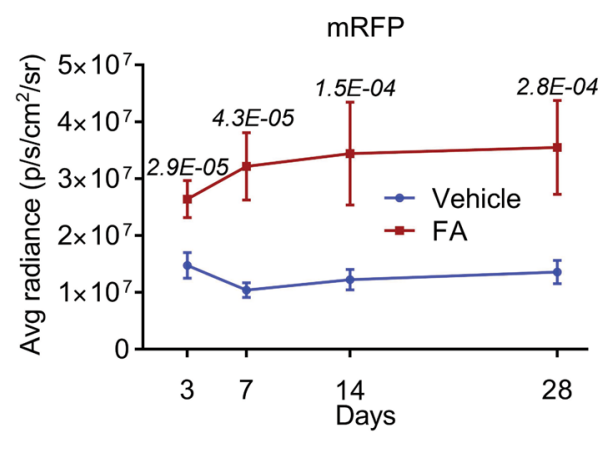

Figure 2. Cellular senescence in kidney tubular cells is an early event after AKI. (A) Confocal microscopy images of p16-3MR-transgenic kidney 14 days after FA injury (left) and vehicle-injected control (right). Scale bars: 2 mm. (B) Confocal microscopy image of a kidney section of p16-3MR-transgenic kidney 14 days after FA injury showing colocalization of mRFP-expressing cells with the proximal tubular marker LTL. Scale bar: $50 \mu \mathrm{m}$. (C) Epifluorescence images of explanted p16-3MR-transgenic kidneys 28 days after FA injury (upper) and control (lower) and (D) quantification at different time points after FA injury. $n=7$ mice per group per time point. All data are presented as mean $\pm \mathrm{SD}$. $P$ values were calculated with 2-tailed Student's $t$ test.

the tubule-specific gene cadherin 16 (Ksp) (38-41), and evaluated the inflammatory components of the SASP. Expression levels of the proinflammatory cytokines IL-1 $\alpha$, IL-1 $\beta$, IL-6, TNF- $\alpha$, and MCP-1 were lower in tubules isolated from ${ }^{\mathrm{Ksp}-\mathrm{Cre}} \mathrm{My} d 88^{\text {I/fl}}$ versus ${ }^{\mathrm{Ksp}-\mathrm{Cre}} \mathrm{My} d 88^{+/+}$(control) mice (Figure 3, A-E), indicating that epithelial TLR/IL-1R signaling promotes the expression of proinflammatory cytokines after injury. Interestingly, the expression of Shh, a ligand that induces proliferation and fibrogenic commitment of mesenchymal progenitor cells (6), was also significantly reduced in the tubules of ${ }^{\mathrm{Ksp}-\mathrm{Cre}} \mathrm{My} d 88^{8 / / f}$ versus control ${ }^{\mathrm{Ksp}-\mathrm{Cre}} \mathrm{Myd} d 88^{+/+}$mice (Figure $3 \mathrm{~F}$ ).

Senescent tubular cells are a source of profibrotic Hh ligands. Shh and its paralog Ihh are ligands in the Hh signaling pathway. The activity of this pathway is required for cell-fate specification during embryonic development, as well as for the homeostasis of some adult organs, regulating the survival and proliferation of tissue progenitors and stem-cell populations (42). During development, the Hh pathway plays critical roles in kidney morphogenesis, with Shh secreted by the ureteric bud epithelium being essential for the survival, proliferation, and differentiation of the metanephric mesenchyme $(43,44)$. After kidney development is complete, no Hh ligands are expressed in the epithelial compartment (45). Because tubular cells in the adult kidney do not express Hh-pathway ligands under normal conditions, we tested the possibility that senescent TECs are the source of these proteins following kidney injury. To this end, we isolated mRFP ${ }^{+}$ and $\mathrm{mRFP}^{-}$cells from the kidneys of $p 16-3 M R$ mice by flow cytometry and used quantitative real-time PCR to measure the expression of Shh and Ihh in each population. Interestingly, the expression of both of these ligands was significantly higher in the $\mathrm{mRFP}^{+}$cells (Figure 4), indicating that senescent TECs are a source of profibrotic Hh-pathway ligands after kidney injury.

Epithelial cell-specific deletion of Myd88 reduces fibrosis and tubular damage after injury. Concordant with the lower expression of cytokines in Myd88-deficient TECs, we also found that kidney fibrosis (Figure 
A

IL-1 $\alpha$
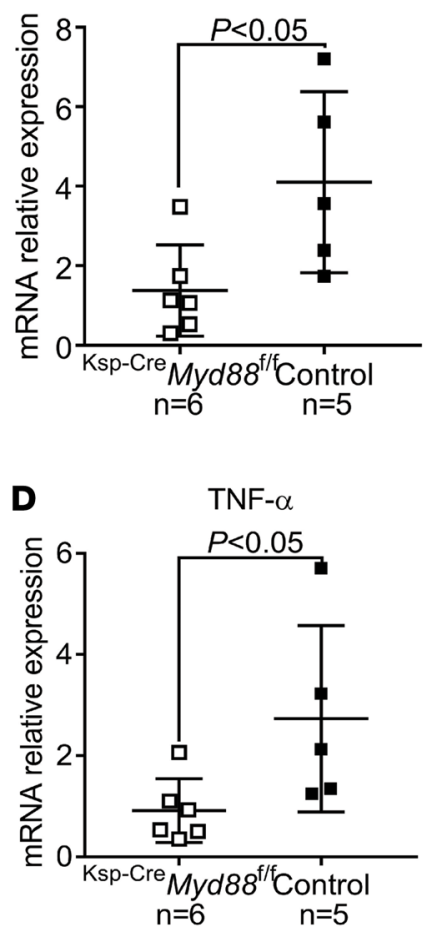

B

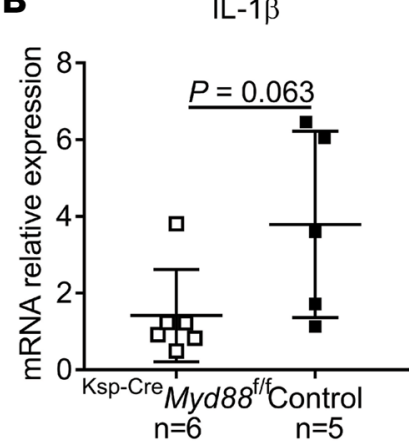

E

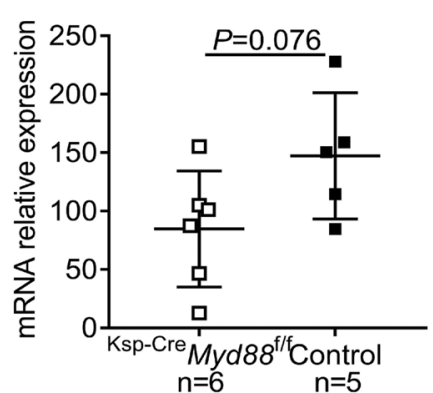

C

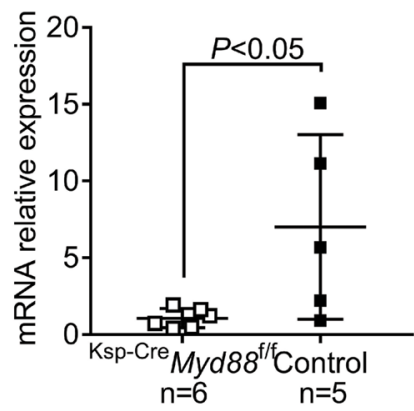

F

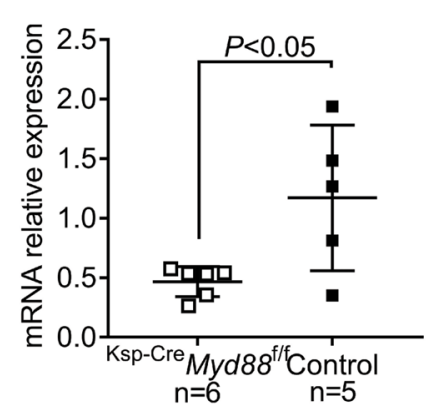

Figure 3. Epithelial cell-specific deletion of Myd88 reduces expression of proinflammatory cytokines after FA injury. (A-E) Expression levels of IL-1 $1 \alpha$, IL-1 $\beta$, IL-6, TNF- $\alpha$, MCP-1, and (F) of the pericyte-activating ligand Shh in isolated tubular cells, measured by real-time quantitative PCR 28 days after FA injury in ${ }^{\mathrm{Ksp}-\text { Cre }} \mathrm{Myd} 88^{f / / 7}$ mice compared with ${ }^{\mathrm{Ksp}-\text {-re }} \mathrm{Myd} 88^{+/+}$controls. The numbers of experimental mice are indicated in the corresponding panels. Data are presented as mean \pm SD. $P$ values were calculated with 2-tailed Student's $t$ test.

$5, \mathrm{~A}-\mathrm{C})$ and interstitial infiltration were lower in ${ }^{\mathrm{Ksp}-\mathrm{Cre}} M y d 88^{8 / / f}$ versus control mice, as estimated by digital analysis of confocal immunofluorescence images obtained using antibodies that recognize markers of fibroblasts (fibroblast-specific protein 1, i.e., FSP1+; Figure 5, D and E), myofibroblasts ( $\alpha$ smooth actin, i.e., $\alpha-\mathrm{SMA}^{+}$; Figure 5, F and G), or macrophages (EGF-like module-containing mucin-like hormone receptor-like 1, i.e., F4/80+; Figure 5, H and I). Kidney damage, quantified based on tubular injury scores of PAS-stained kidney sections assigned by a pathologist, was also significantly lower in

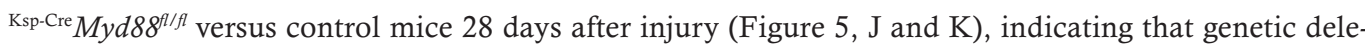
tion of Myd88 before FA-induced injury is sufficient to protect kidneys from damage. This conclusion was also supported by the reduced mortality of ${ }^{\mathrm{Ksp}-\mathrm{Cre}} M y d 88^{f / / f l}$ but not control mice during the first 10 days after injury (Figure 5L). This effect on mortality is likely a consequence of injury being milder in the context of the Myd88 deficiency, as suggested by the lower levels of serum creatinine 2 days after FA administration (Figure 5M).

Epithelial cell-specific deletion of Myd88 reduces tubular cell senescence after injury. Given that TLR/IL-1dependent activation of NF-кB is responsible for the paracrine effect of senescent cells (14) and senescent cells are able to induce secondary senescence in neighboring cells, we asked whether epithelial inactivation of Myd88 suppresses the spread of kidney senescence. Indeed, this was the case: tubular deletion of Myd88 led to a reduction in SA- $\beta-$ Gal activity (Figure 6, A and B), as well as to increases in the abundance of nuclear LAMNB1 (Figure 6, C and D) and proliferating tubular cells (Figure 6, E and F) 28 days after injury. These results indicate that epithelial TLR/IL-1R signaling controls the onset of tubular senescence, and that the inhibition of this signaling is sufficient to limit the accumulation of senescent tubular cells after kidney injury.

Inhibition of epithelial innate immunity after injury reduces tubular senescence and fibrosis but does not protect from tubular damage. Because reduced fibrosis, damage, and senescence in constitutive Myd88-knockout mice could be due to less severe injury ab initio, as suggested by the lower serum creatinine levels in

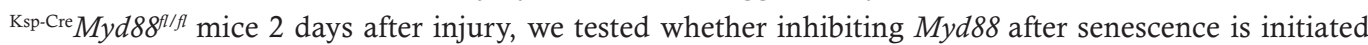
also limits the accumulation of tubular senescent cells. To this end, we used the FA model of injury in 
A

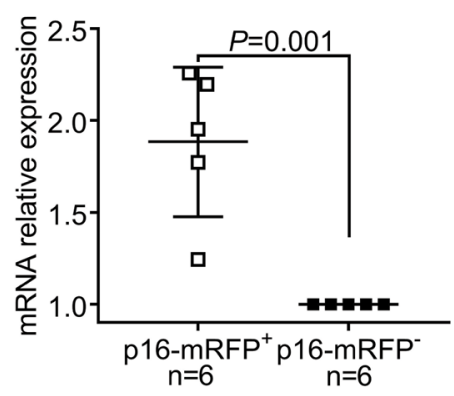

B

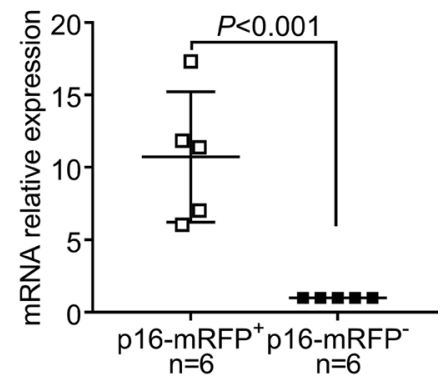

Figure 4. Senescent tubular cells are a source of profibrotic Hedgehog ligands. (A and B) Relative expression enrichment of Shh and Ihh in mRFP+ cells compared with mRFP- cells from the same kidneys 14 days after FA injury. The numbers of experimental mice are indicated in the corresponding panels. Data are presented as mean $\pm \mathrm{SD}$. $P$ values were calculated with 2-tailed Student's $t$ test.

${ }_{\text {KspCre-ERT2 }} M y d 88^{R / f}$ mice, in which Cre expression driven by the cadherin 16 promoter is induced upon tamoxifen injection (46). We activated Cre expression 5 days after FA injection, that is, after senescence was detectable in injured kidneys (Figure 2D and Supplemental Figure 1), and looked for the presence of senescent cells 28 days after injury. Deletion of $M y d 88$ in tubular cells beginning 5 days after injury (Figure 7A) reduced the activity of SA- $\beta-$ Gal (Figure 7, B and C) and increased the abundance of both nuclear LAMNB1 (Figure 7, D and E) and proliferating tubular cells (Figure 7, F and G). Consistent with these findings, interstitial infiltration and fibrosis were also decreased in ${ }^{\mathrm{Ksp} C \mathrm{Cre}-\mathrm{ERT} 2} \mathrm{My} d 88^{\text {/I/f }}$ versus control mice (Figure 8, A-I). However, Myd88 deletion did not confer a significant protective effect with respect to tubular damage (Figure 8, J and K), suggesting that inhibition of TLR/IL-1R signaling is effective at limiting senescence and fibrosis, but not tubular damage, after injury has occurred.

Genetic and pharmacologic elimination of senescent cells after FA injury partially protects against fibrosis but does not affect tubular damage. The above-described experiments in FA-injured KspCre-ERT2 Myd88 ${ }^{A / t}$ mice indicate that limiting the tubule-derived SASP is sufficient to prevent kidney inflammation and fibrosis after FA injury. To test whether the elimination of senescent cells from kidneys after injury prevents the progression of kidney damage and fibrosis, we selectively induced apoptosis in HSV-TK-p16 $6^{\text {InktA }}$ expressing senescent cells of $p 16-3 M R$ mice 5 days after FA injury, injecting GCV daily for 10 days (Figure 9A) (17). An increase in the number of apoptotic cells (positive for activated caspase- 3 and TUNEL staining) 10 days after the first GCV injection confirmed that cell death was efficiently induced (Supplemental Figure 2, A-D). Consistent with these findings, TEC senescence (assessed based on SA- $\beta-$ Gal activity and nuclear LAMNB1 abundance) declined significantly (Supplemental Figure 2, E-H) and cell proliferation increased (Supplemental Figure 2, I and J) 28 days after injury. In addition, fibrosis (Figure 9, B-D), as well as interstitial infiltration of fibroblasts (FSP-1+; Figure 9, E and F), macrophages $\left(\mathrm{F} 4 / 80^{+}\right.$; Figure $9, \mathrm{G}$ and $\left.\mathrm{H}\right)$, and myofibroblasts ( $\alpha$-SMA ${ }^{+}$; Figure 9 , I and J), were reduced in GCV-treated versus vehicle-treated (control) mice. However, no significant difference in tubular damage was observed (Figure 9, K and L).

Given the apparent discrepancy between the effects on fibrosis and tubular damage in the above-described experiments, we eliminated senescent cells using a second approach, applying the synthetic peptide FOXO4-DRI, which induces apoptosis of senescent cells by disrupting the interaction between FOXO4 and p53 (Supplemental Figure 3A) $(24,47)$. FOXO4-DRI was as effective as GCV in both inducing apoptosis (Supplemental Figure 3, B-E) and reducing the number of senescent cells (Supplemental Figure 3, F-K) within 28 days of injury. However, treatment with FOXO4-DRI did not lead to reduced interstitial fibrosis (Supplemental Figure 4, A-C), cellular infiltration generally (reduction of only the number of FSP1 ${ }^{+}$fibroblasts) (Supplemental Figure 4, D-I), or tubular damage (Supplemental Figure 4, J and $\mathrm{K}$ ). Overall, these results suggest that eliminating senescent cells at early stages after injury may have beneficial effects with regard to inflammation and fibrosis, but that this does not protect against tubular damage regardless of whether the actions are early or late. They also suggest that GCV and FOXO4-DRI might target distinct subpopulations of senescent cells. 
A

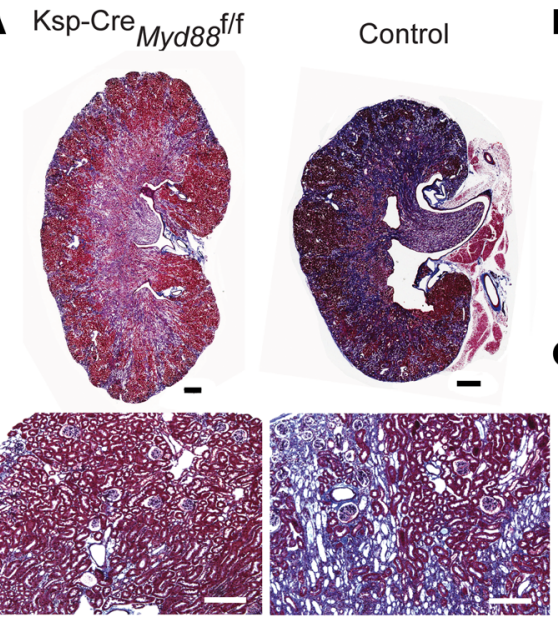

D $\mathrm{Ksp}-\mathrm{Cre}_{\mathrm{Myd} 88^{\mathrm{f} / \mathrm{f}}}$

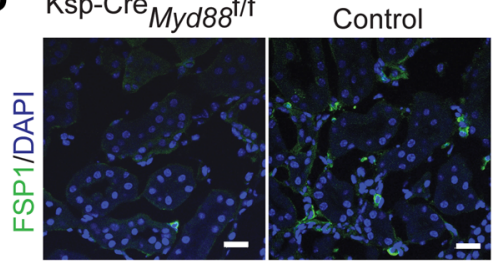

$\mathbf{F}$

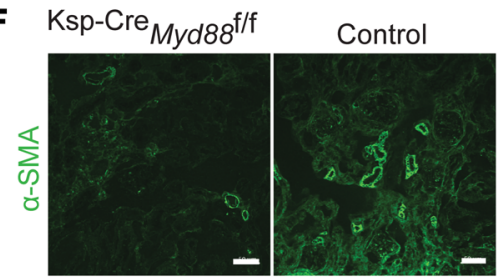

H

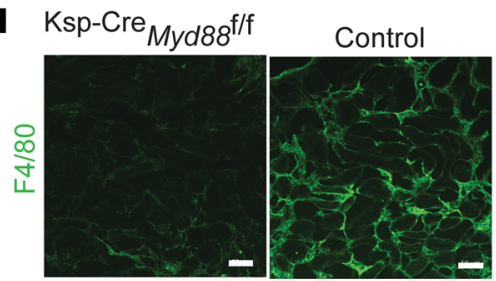

B

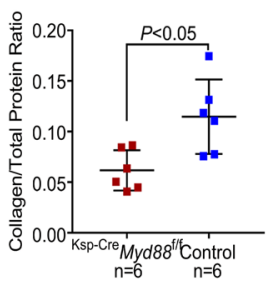

C

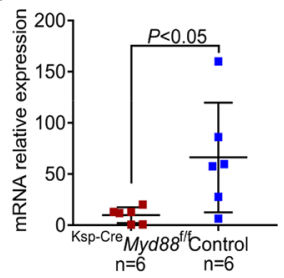

E

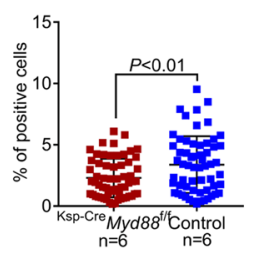

G
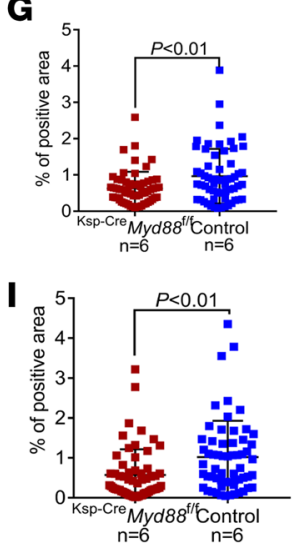

J

Ksp-Cre ${ }_{M y d 88^{f / f}}$
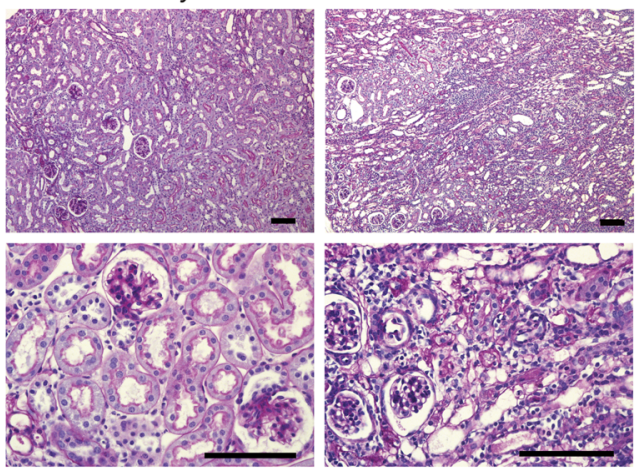

K

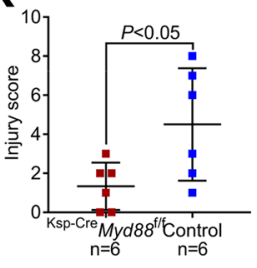

$\mathbf{L}$

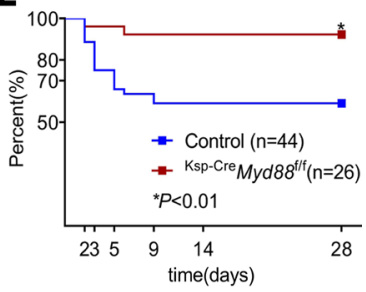

M

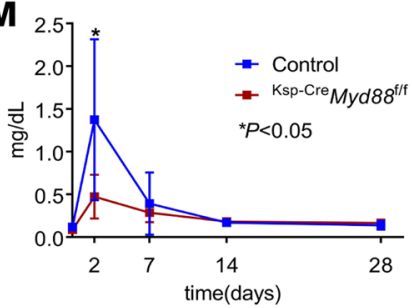

Figure 5. Epithelial cell-specific deletion of Myd88 reduces kidney damage and fibrosis. (A) Representative images of trichrome-stained kidneys at low

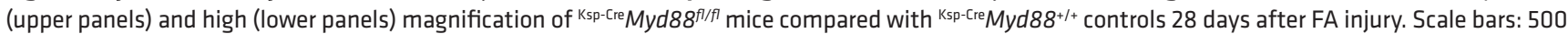
$\mu \mathrm{m}$ (upper) and $200 \mu \mathrm{m}$ (lower). (B) Collagen/total protein content ratios and (C) real-time quantitative PCR of the collagen 1 mRNA levels in kidney cortexes

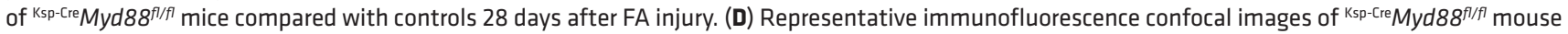
kidneys immunostained for the fibroblast marker FSP1 compared with controls 28 days after FA injury, and (E) corresponding quantification by digital image analysis. Scale bars: $20 \mu \mathrm{m}$. (F) Representative immunofluorescence confocal images of Ksp-cre Myd88//f/ mouse kidney immunostained for the myofibroblast marker $\alpha$-SMA compared with controls 28 days after FA injury, and (G) corresponding quantification by digital image analysis. Scale bars: $20 \mu \mathrm{m}$. (H) Representative immunofluorescence confocal images of Ksp-Cre $M y d 88^{f / f l}$ mouse kidneys immunostained for the macrophage marker F4/80 compared with controls 28 days after FA injury, and (I) corresponding quantification by digital image analysis. Scale bars: $20 \mu \mathrm{m}$. (J) Representative images of PAS-stained kidneys at low (upper panels) and high (lower panels) magnification of Ksp-Cre Myd8 8 f//fl mice compared with controls 28 days after FA injury. Scale bars: $500 \mu \mathrm{m}$ (upper) and $200 \mu \mathrm{m}$ (lower). (K) Tubular injury scores in Ksp-Cre Myd88f/f mice compared with controls 28 days after FA injury. Experimental numbers of mice are reported in each panel. (L) Kaplan-Meier curves of ${ }^{\mathrm{Ksp}-\mathrm{Cre}} \mathrm{Myd} 88^{\mathrm{fl} / \mathrm{fl}}$ mice compared with controls. Numbers of experimental mice indicated in the panel. (M)

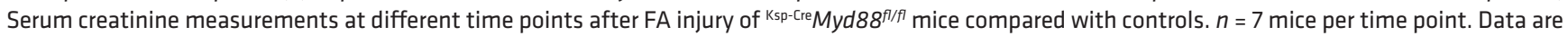
presented as mean \pm SD. $P$ values were calculated using the 2-tailed Student's $t$ test.

\section{Discussion}

Here, we report on our examination of the occurrence of cellular senescence in mice after kidney injury and whether senescence contributes to the progression of tubular damage and fibrosis after AKI. The positivity for markers of cellular senescence in 3 distinct models of kidney injury indicates that cellular senescence is a common event, irrespective of the type of injury. Using a transgenic reporter to mark senescent cells, we 
A
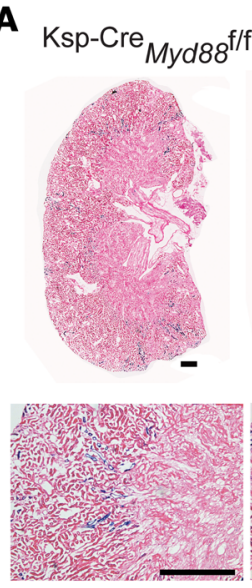

B
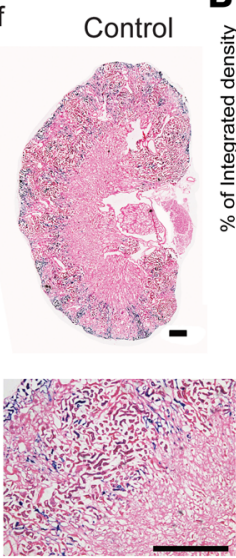

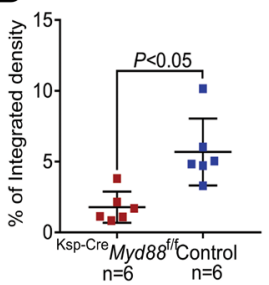

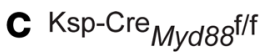

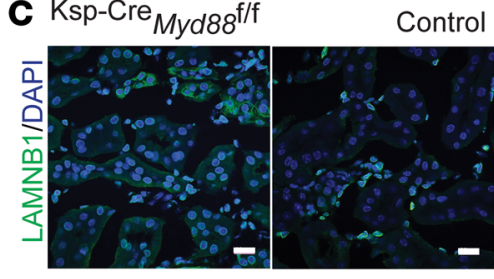

Control
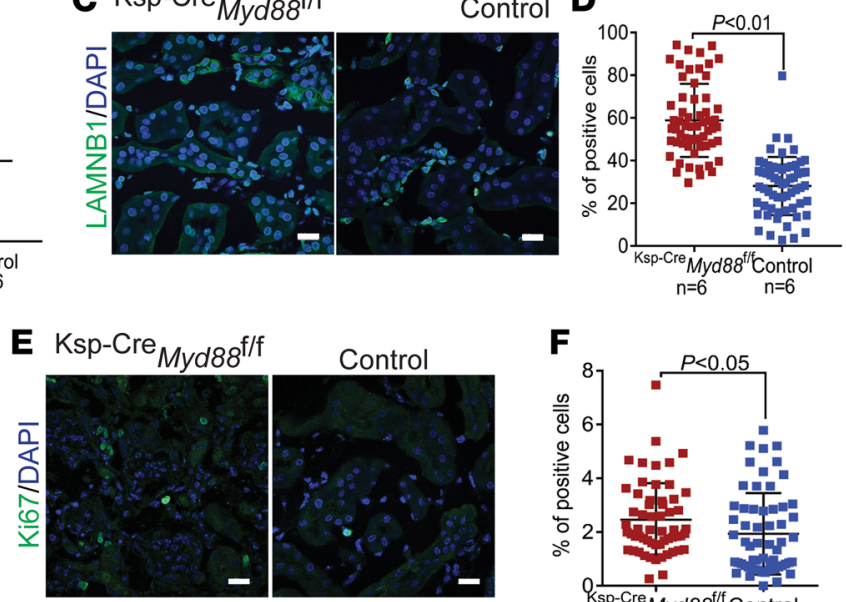

$\mathbf{F}$

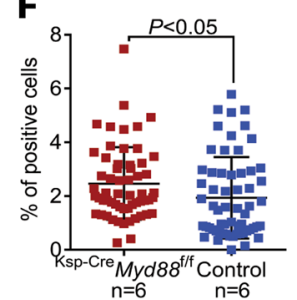

Figure 6. Epithelial cell-specific deletion of Myd88 reduces senescence in tubular cells. (A) Representative images of kidneys stained for SA- $\beta$-Cal activity at low (upper panels) and high (lower panels) magnification of Ksp-Cre Myd8 $88^{f / f l}$ mice compared with Ksp-Cre $M y d 88^{+/+}$controls 28 days after FA injury, and (B) corresponding quantification by digital image analysis. Scale bars: $500 \mu \mathrm{m}$ (upper) and $200 \mu \mathrm{m}$ (lower). (C) Representative immunofluorescence confocal images of Ksp-Cre Myd8 $88^{f / f l}$ mouse kidneys probed with an antibody against LAMNB1 compared with controls 28 days after FA injury, and (D) corresponding quantification. Scale bars: $20 \mu \mathrm{m}$. (E) Representative immunofluorescence confocal images of Ksp-Cre Myd88 8 f/fl mouse kidneys probed with an antibody against the proliferative marker Ki67 compared with controls 28 days after FA injury, and (F) corresponding quantification. Scale bars: $20 \mu \mathrm{m}$. Numbers of experimental mice are reported in each panel. Ten images per mouse. Data are presented as mean \pm SD. $P$ values were calculated with 2-tailed Student's $t$ test.

showed, based on the localization and morphology of this marker, that senescence occurs mostly in cells of the proximal tubule. An interesting and unexpected finding was that tubular cell senescence occurs relatively early, independent of the type of injury, and that the senescent-cell burden slowly increases over time. This observation may be of clinical relevance, because it suggests that any attempt to limit the number of cells undergoing senescence after kidney injury should be carried out within a few days of the pathogenic event, earlier than the onset of fibrosis and even earlier than the appearance of clinical signs of CKD.

TLRs are pattern recognition receptors that respond to pathogen-associated or tissue damage-associated molecules, and they can induce transcription of inflammatory cytokines and chemokines in pathological situations that are potentially dangerous to the host (48). TLRs are most abundant in lympho-monocytes but are also present in parenchymal and epithelial cells, including kidney tubular cells (48). The contribution of TLR signaling to inflammation and fibrosis after kidney injury has been reported in studies performed in mice that are constitutively null for TLR2 and TLR4 and, more recently, in mice with pericyte-specific deletion of components of the TLR pathway $(34,49,50)$. However, the function of this signaling axis within the epithelial compartment has not to our knowledge been investigated. The results of our experiments indicate that signaling downstream of TLR/IL-1R in the epithelium not only mediates the contribution of tubule-derived proinflammatory cytokines after kidney injury, a mechanism that has not previously been described, but is also a key factor in the onset of senescence in tubular cells and its spread to the surrounding epithelium. This latter finding is potentially important because early therapeutic interventions targeting the epithelial TLR/IL-R1 signaling axis could limit the autocrine and paracrine reinforcing effects of the SASP. Although further studies will be required to understand the details of the molecular mechanisms by which innate immunity governs the cellular programs that lead to senescence, this study reveals what we believe is a novel function of TLR/IL-R1 signaling in controlling the onset of TEC senescence in a cell-autonomous manner, and the proliferation and cell fate of pericytes non-cell autonomously, consistent with the concept that the tubular epithelium triggers kidney disease following injury and also drives its progression.

Our findings also highlight the limited efficacy of senolytic drugs, which induce the apoptosis of senescent cells to limit kidney damage. Our experiments with $p 16-3 M R$-transgenic mice and the FOXO4-DRI peptide yielded different results in terms of protection from fibrosis. These findings suggest that distinct types of senescent cells may coexist, and that subpopulations of these cells may have diverse functions in injured tissues. Importantly, in both sets of experiments, eliminating senescent cells, at least at early stages 
A

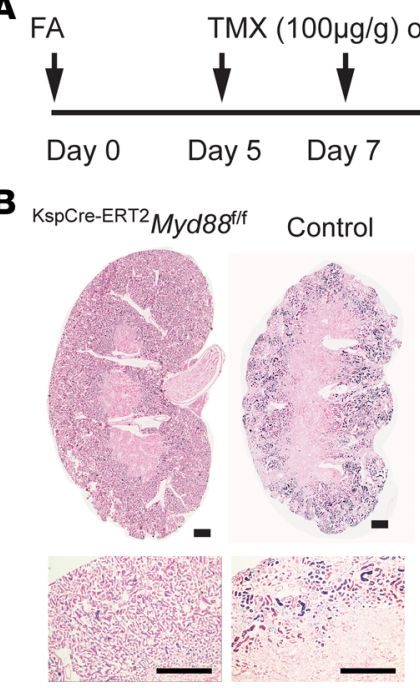

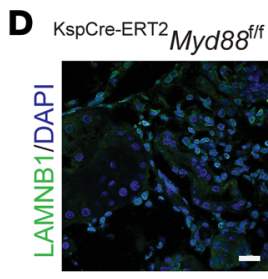
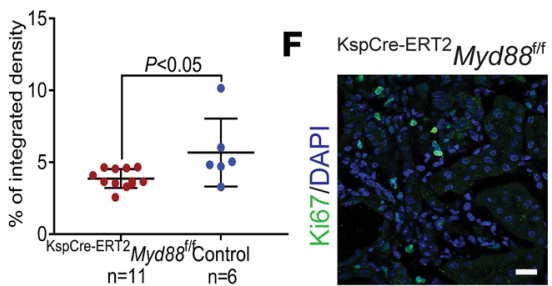

$\mathbf{E}$
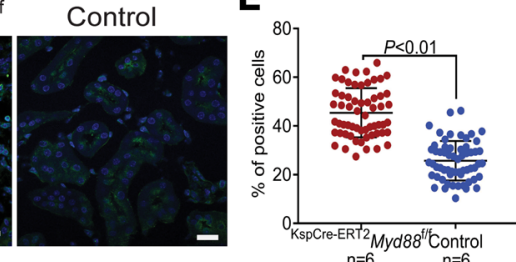

$\mathrm{n}=6$

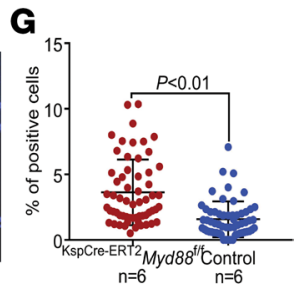

Figure 7. Epithelial cell-specific deletion of Myd88 after injury reduces senescence in tubular cells. (A) Schematic representation of the protocol for

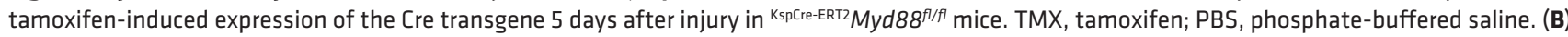
Representative images of kidneys stained for SA- $\beta$-Gal activity at low (upper panels) and high (lower panels) magnification of Kspcre-ERT2 Myd88 ${ }^{f / / f l}$ mice compared with controls ( ${ }^{\text {KspCre-ERT2 }} \mathrm{Myd} 88^{+/+}$) 28 days after FA injury, and (C) corresponding digital image analysis. Scale bars: $500 \mu \mathrm{m}$ (upper) and $200 \mu \mathrm{m}$ (lower). (D) Representative immunofluorescence confocal images of KspCre-ERT2Myd88 f/f/ mouse kidneys probed with an antibody against LAMNB1 compared with controls 28 days after FA injury, and (E) corresponding quantification. Scale bars: $20 \mu \mathrm{m}$. (F) Representative immunofluorescence confocal images of KspCre-ERT2 Myd8 $88^{f / f l}$ mouse kidneys probed with an antibody against the proliferation marker Ki67 compared with controls 28 days after FA injury, and (G) corresponding quantification. Scale bars: $20 \mu \mathrm{m}$. Numbers of experimental mice are reported in each panel. Ten images per mouse. Data are presented as mean \pm SD. $P$ values were calculated with 2-tailed Student's $t$ test.

after injury, did not affect kidney damage and repair. This is in contrast to what has been reported in the context of natural or pathologic aging, where clearing of senescent cells improves tissue homeostasis (47, 51-54). Currently, the causes of these different outcomes are not clear. One possibility, given the higher burden of senescent cells in the context of acute injury, is that widespread induction of apoptosis in tubular cells may offset the benefits of reducing proinflammatory stimuli like those derived from slowly accumulating senescent cells in aging tissues, resulting in an increase in tubular damage and the onset of maladaptive processes. However, it is also possible that the concomitant elimination of tubular and nonepithelial senescent cells by senolytic drugs compromises proreparative processes after injury (24).

In conclusion, we have identified tubular cell senescence as an early central mechanism that leads to further accumulation of senescent cells after kidney injury and drives the progression of kidney damage and fibrosis through both cell-autonomous activation of innate immune signaling and non-cell-autonomous control of pericyte fate. Importantly, the partial effectiveness of eliminating senescent cells after injury with respect to reducing inflammation and fibrosis, but not tubular damage, suggests that cellular senescence may also contribute to tissue repair after AKI.

\section{Methods}

Mouse kidney injury. All mice were on a C57BL/6J background. Myd88 ${ }^{\mathrm{fl}}$ mice (Jax 008888) (55) were obtained from the Jackson Laboratory and crossed with Ksp-Cre (56) or KspCre-ERT2 (57) transgenic mice, obtained from the O'Brian Kidney Center Animal Models Core of UT Southwestern Medical Center. The phenotypes of the ${ }^{\mathrm{Ksp}-\mathrm{Cre}} M y d 88^{f / f l}$ and ${ }^{\mathrm{KspCre}-\mathrm{ERT} 2} M y d 88^{f l / f l}$ mice were compared to those of controls ( ${ }^{\mathrm{Ksp}-\mathrm{Cre}} \mathrm{Myd} 88^{+/+}$and ${ }^{\mathrm{KspCre}-\mathrm{ERT} 2} \mathrm{Myd88^{+/+ }}$ ) (58). p16-3MR mice were from the Buck Institute for Research on Aging. Male mice 6 to 8 weeks of age were injected intraperitoneally with FA (Sigma-Aldrich) at a single dose of $250 \mu \mathrm{g} / \mathrm{g}$ in $0.3 \mathrm{mM} \mathrm{NaHCO}$, solution and sacrificed at various times thereafter.

Isolation of renal tubular cells. Kidneys were decapsulated and dissected, and cortexes were minced.

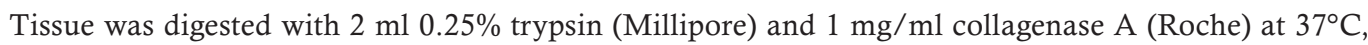
$5 \% \mathrm{CO}_{2}$ for 20 minutes. Tissue lysates were diluted with PBS, and passed through $100-\mu \mathrm{m}$ and $70-\mu \mathrm{m}$ strainers. The cells that did not pass through the $70-\mu \mathrm{m}$ strainer were collected, centrifuged, and resuspended with $0.5 \mathrm{ml} 0.25 \%$ trypsin. After 5 minutes of digestion at $37^{\circ} \mathrm{C}$, cell suspensions were passed 
A

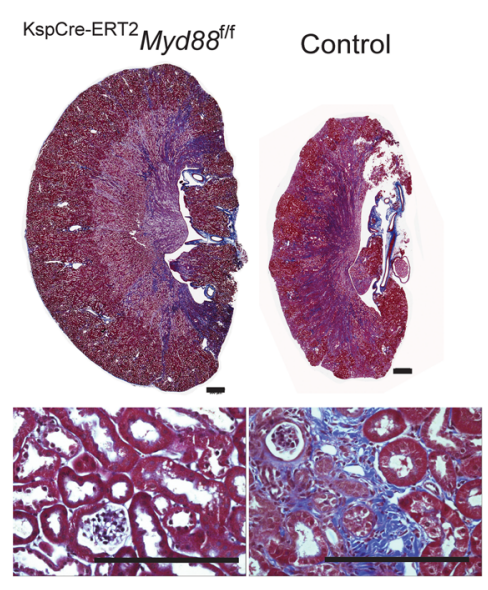

B

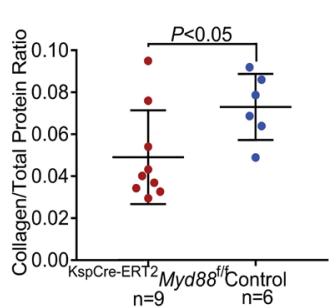

D

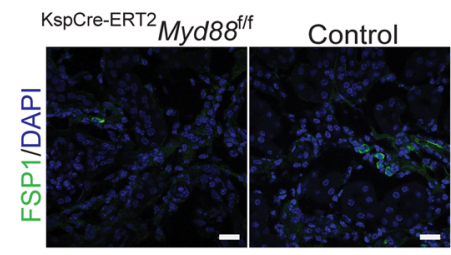

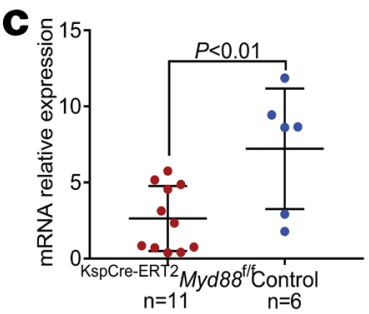

E

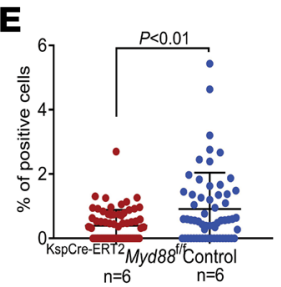

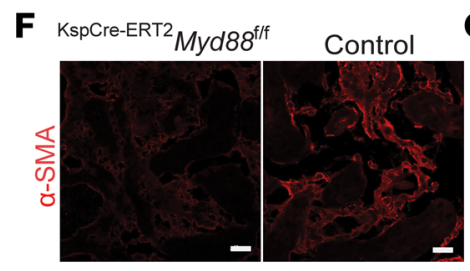

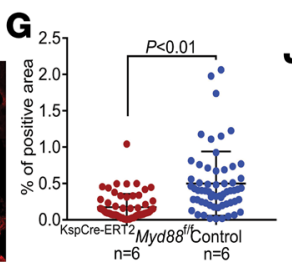

J
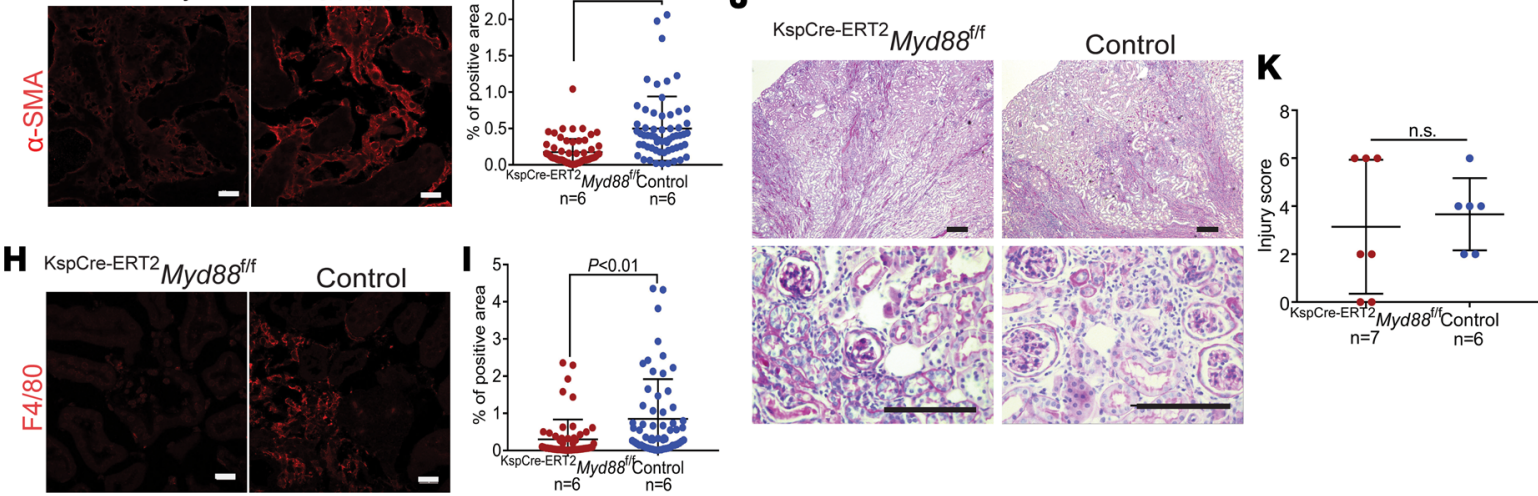

Figure 8. Epithelial cell-specific deletion of Myd88 after injury reduces fibrosis but does not protect from tubular damage. (A) Representative images

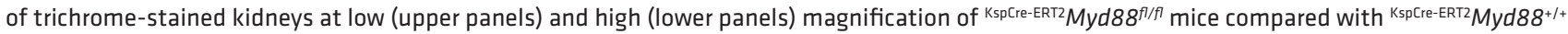
controls 28 days after FA injury. Scale bars: $500 \mu \mathrm{m}$ (upper) and $200 \mu \mathrm{m}$ (lower). (B) Collagen/total protein content ratios and (C) real-time quantitative PCR of the collagen 1 mRNA levels in kidney cortexes of KspCre-ERT2 Myd8 $88^{f / f l}$ mice compared with controls 28 days after FA injury. (D) Representative immunofluorescence confocal images of ${ }^{\mathrm{KspC} C \mathrm{e}-\mathrm{ERT} 2} \mathrm{Myd} 88^{\mathrm{f} / \mathrm{f}}$ mouse kidneys immunostained for the fibroblast marker FSP1 compared with controls 28 days after FA injury, and (E) corresponding quantification by digital image analysis. Scale bars: $20 \mu \mathrm{m}$. (F) Representative immunofluorescence confocal images of KspCre-ERT2 Myd88 ${ }^{f / / f l}$ mouse kidneys immunostained for the myofibroblast marker $\alpha$-SMA compared with controls 28 days after FA injury, and (C) corresponding quantification by digital image analysis. Scale bars: $20 \mu \mathrm{m}$. (H) Representative immunofluorescence confocal images of Kspcre-ERT2 Myd88 $8^{f / f l}$ mouse kidneys immunostained for the macrophage marker F4/80 compared with controls 28 days after FA injury and (I) corresponding quantification by digital image analysis. Scale bars are $20 \mu \mathrm{m}$. (J) Representative images of PAS-stained kidneys at low (upper panels) and high (lower panels) magnification of KspCre-ERT2 Myd8 $88^{f / f l}$ mice compared with controls 28 days after FA injury. Scale bars: $500 \mu \mathrm{m}$ (upper) and $200 \mu \mathrm{m}$ (lower). (K) Tubular injury scores for ${ }^{\mathrm{KspCre}-\mathrm{ERT} 2}$ Myd $88^{f / / f}$ mice compared with controls 28 days after FA injury. Experimental numbers of mice are reported in each panel. Data are presented as mean \pm SD. $P$ values were calculated using the 2 -tailed Student's $t$ test.

through a $40-\mu \mathrm{m}$ strainer and centrifuged. Single cells were harvested and resuspended in DMEM/F12 medium and incubated at $37^{\circ} \mathrm{C}, 5 \% \mathrm{CO}_{2}$.

Fluorescence-activated cell sorting. Kidney cortexes were dissected and digested for 30 minutes with 10 $\mathrm{mg} / \mathrm{ml}$ Bacillus licheniformins protease (Sigma-Aldrich) and $125 \mathrm{U} / \mathrm{ml}$ DNAse (Worthington) on ice with pipetting every 5 minutes. Sorting of live $\mathrm{RFP}^{+}$and $\mathrm{RFP}^{-}$cells was carried out using the FACSAria II system (BD) at the University of Iowa FACS Facility.

Ratio of collagen to total protein. Ten paraffin-embedded kidney sections ( $10 \mu \mathrm{m}$ thickness) per mouse were hydrolyzed in $150 \mathrm{ml} 6 \mathrm{M} \mathrm{HCl}$, incubated for 20 hours at $95^{\circ} \mathrm{C}$, and centrifuged for 10 minutes at $16,000 \mathrm{~g}$ after cooling. Total collagen and protein concentrations in the supernatant were determined using collagen and protein assay kits from QuickZyme. The collagen to total protein ratio of each sample was calculated with these 2 values.

Antibodies. $\alpha$-SMA (A5228) antibody was from Sigma-Aldrich. F4/80 (14-4801-82), cleaved caspase-3 (AB3623MI), and p16 ${ }^{\text {INK4a }}$ (MA5-17093) antibodies were from Thermo Fisher Scientific. FSP1 (ab27957), Ki67 (ab15580), and LAMNB1 (ab16048) antibodies were from Abcam. 
A FA

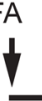

Day 0
$\operatorname{GCV}(25 \mu \mathrm{g} / \mathrm{g} / \mathrm{day}) / \mathrm{PBS}$

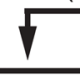

Day 5

C

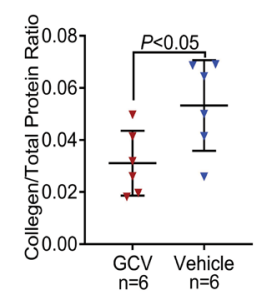

E
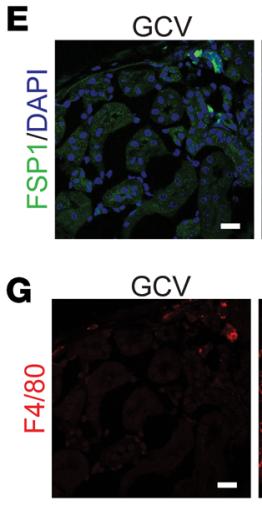

I

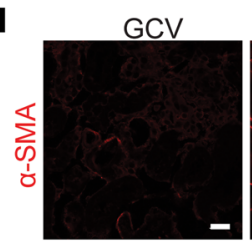

D

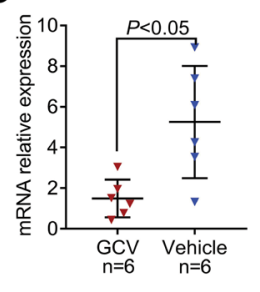

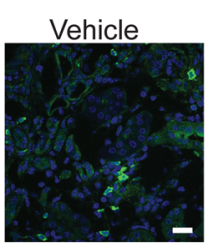

$F$

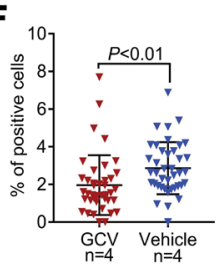

K

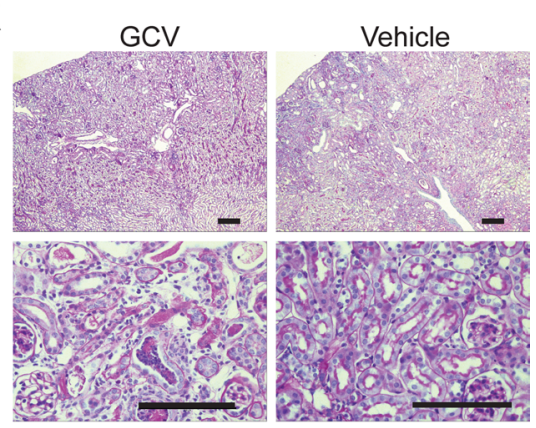

B
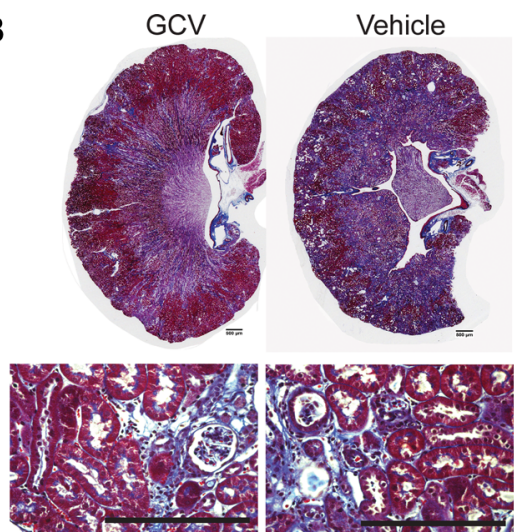

Vehicle
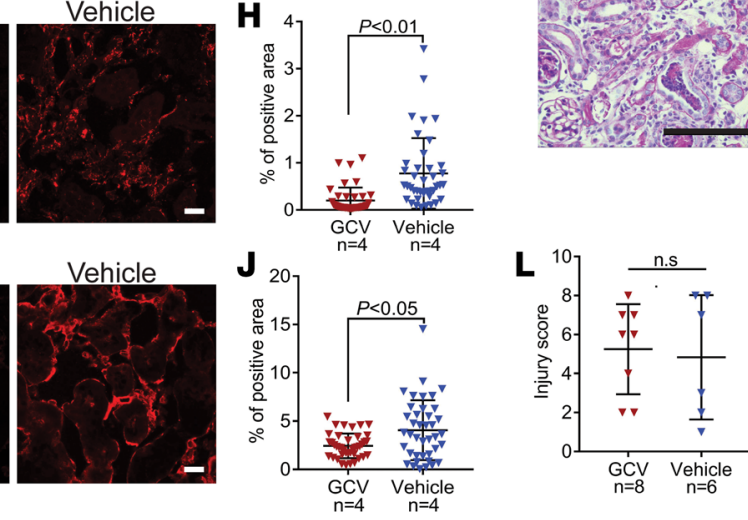

Figure 9. Genetic clearance of senescent cells ameliorates interstitial fibrosis but not tubular damage after AKI. (A) Schematic representation of the protocol for inducing clearance of senescent cells by ganciclovir administration to FA-injured p16-3MR mice. GCV, ganciclovir; PBS, phosphate-buffered saline. (B) Representative images of trichrome-stained kidneys at low (upper panels) and high (lower panels) magnification of GCVtreated mice compared with PBS-treated controls 28 days after FA injury. Scale bars: $500 \mu \mathrm{m}$ (upper) and 200 $\mu \mathrm{m}$ (lower). (C) Collagen/total protein content ratios and (D) real-time quantitative PCR of the collagen 1 mRNA levels in kidney cortexes of GCV-treated and vehicle-treated mice 28 days after FA injury. (E) Representative immunofluorescence confocal images of GCV-treated mouse kidneys immunostained for the fibroblast marker FSP1 compared with controls 28 days after FA injury, and (F) corresponding quantification by digital image analysis. Scale bars: $20 \mu \mathrm{m}$. (G) Representative immunofluorescence confocal images of GCV-treated mouse kidneys immunostained for the macrophage marker F4/80 compared with controls 28 days after FA injury, and (H) corresponding quantification by digital image analysis. Scale bars: $20 \mu \mathrm{m}$. (I) Representative immunofluorescence confocal images of GCV-treated mouse kidneys immunostained for the myofibroblast marker $\alpha$-SMA compared with controls 28 days after FA injury, and (J) corresponding quantification by digital image analysis. Scale bars: $20 \mu \mathrm{m}$. (K) Representative images of PAS-stained kidneys at low (upper panels) and high (lower panels) magnification of GCV-treated mice compared with controls 28 days after FA injury. Scale bars: $500 \mu \mathrm{m}$ (upper) and $200 \mu \mathrm{m}$ (lower). (L) Tubular injury scores of GCV-treated mice compared with controls 28 days after FA injury. Experimental numbers of mice are reported in each panel. Data are presented as mean \pm SD. $P$ values were calculated with 2-tailed Student's $t$ test.

Immunofluorescence staining. Tissues were collected after perfusion with PBS and 4\% paraformaldehyde (PFA), then fixed in $4 \%$ PFA at $4^{\circ} \mathrm{C}$ overnight, left in $18 \%$ sucrose in PBS at $4^{\circ} \mathrm{C}$ overnight, and embedded in optimal cutting temperature (OCT) compound. Frozen tissue sections $(10 \mu \mathrm{m})$ were air dried for $30 \mathrm{~min}-$ utes at room temperature (RT) and sequentially incubated with $0.1 \%$ Triton X-100 solution for 20 minutes, $0.1 \%$ sodium borohydride $\left(\mathrm{NaBH}_{4}\right)$ solution for 30 minutes, and blocking solution $(10 \%$ goat serum, $0.1 \%$ bovine serum albumin in PBS) for 1 hour at RT. Tissues were incubated with primary antibody diluted in 
blocking solution overnight at $4^{\circ} \mathrm{C}$, and with fluorescently labeled secondary antibody for 1 hour at RT. Samples were mounted in ProLong Gold Antifade Mountant with 4',6-diamidino-2-phenylindole (DAPI) (Thermo Fisher Scientific). Images were acquired using a Zeiss Axioplan 2 deconvolution microscope or a Zeiss LSM 710 confocal microscope with constant acquisition parameters.

$S A-\beta$-Gal staining. Kidneys were fixed in $4 \%$ PFA and incubated in $18 \%$ sucrose as previously described (59). Frozen sections $(8 \mu \mathrm{m})$ were air dried for 20 minutes and then incubated at $37^{\circ} \mathrm{C}$ for 12 to 16 hours in fresh SA- $\beta$-Gal staining solution with $1 \mathrm{mg} / \mathrm{ml} \mathrm{X-Gal} \mathrm{(Teknova)} \mathrm{in} \mathrm{dimethylformamide,} 40 \mathrm{mM}$ citric acid/ sodium phosphate ( $\mathrm{pH}$ 6.0), $5 \mathrm{mM}$ potassium ferrocyanide, $150 \mathrm{mM} \mathrm{NaCl}$, and $2 \mathrm{mM} \mathrm{MgCl}$. Cells were counterstained with eosin and imaged by bright-field microscopy.

TUNEL assay. Tissues were collected after perfusion with PBS, and then embedded in OCT compound. Frozen tissue sections $(8 \mu \mathrm{m})$ were air dried for 30 minutes, incubated with $4 \%$ PFA for 20 minutes at RT, permeabilized with $0.1 \%$ Triton X-100 in $0.1 \%$ sodium citrate solution, and stained using the quick protocol of the Fluorometric TUNEL System (Promega). Images were acquired using a Zeiss Axioplan 2 deconvolution microscope.

Digital image analysis. Kidney sections stained with SA- $\beta-G a l$, Masson's trichrome, and immunofluorescence were used for digital image quantification of each respective signal. For each cortical region, 10 consecutive $\times 200$ fields per section were selected for measurement. Signals were quantified by digital elaboration of microscopy images using ImageJ software (NIH; http://rsb.info.nih. gov/ij/).

Tubular injury score. Whole kidneys were stained with periodic acid-Schiff (PAS) by the pathology core at UT Southwestern Medical Center or the University of Iowa, and were imaged using a Zeiss Axioplan 2 deconvolution microscope. Tubular injury scores were assigned to PAS-stained kidney sections $(4 \mu \mathrm{m})$ by an experienced kidney pathologist who was blinded to the identity of the samples. We adopted published criteria as described for each parameter (60). Tubular injury/degeneration was defined as including vacuolization, luminal cell casts, and acellular/atrophic changes, and scoring was as follows: 0 = none detected; $1=1 \%-10 \%$ tubules involved; $2=10 \%-25 \%$ tubules involved; $3=25 \%-50 \%$ tubules involved; $4=$ greater than $50 \%$ tubules involved. Tubulointerstitial inflammation was defined as the presence of lymphocytes in perivascular and interstitial cortical areas, and scoring was as follows: $0=$ no significant inflammation; $1=$ $1 \%-10 \%$ foci in perivascular areas; $2=10 \%-25 \%$ of cortex involved; $3=25 \%-50 \%$ of cortex involved; $4=$ greater than $50 \%$ of cortex involved.

Quantitative real-time PCR. Total RNA was extracted using the RNeasy Mini Kit (Qiagen). RNA was reverse transcribed using the iScript cDNA Synthesis Kit (Bio-Rad). Real-time PCR was performed using the CFX Connect Real-Time PCR Detection System (Bio-Rad) and iTaq Universal SYBR Green Supermix (Bio-Rad). All real-time PCR experiments were performed in triplicate. The sequences of PCR primers (IDT) are provided in Supplemental Table 1.

Statistics. All quantitative data are presented as mean $\pm \mathrm{SD}$. Statistical significance (a $P$ value $\leq 0.05$ was considered significant) was calculated using the 2-tailed unpaired Student's $t$ test for 2 groups, or ANOVA and Tukey's multiple comparisons test for more than 2 groups, using SPSS 17.0 software (IBM) or Prism 7 (GraphPad Software).

Study approval. All procedures were approved by the Institutional Animal Care and Use Committee at the University of Iowa (protocol 7021970).

\section{Author contributions}

HJ performed experiments, analyzed data, prepared figures, and contributed to writing of the manuscript. YZ, QD, SSW, and D. Lu performed experiments and analyzed data. AW, D. Liu, MP, CR, CC, and SE performed experiments. DFD and PR analyzed data. DZO participated in writing of the manuscript. $\mathrm{MCH}$, $\mathrm{DZO}, \mathrm{YC}$, and JC provided reagents. MA designed experiments, analyzed data, prepared figures, and contributed to writing of the manuscript.

\section{Acknowledgments}

This work was supported by 2 NIH grants (1R01DK090326 and P30DK079328 to MA). The data presented were obtained at the Flow Cytometry Facility, which is a Carver College of Medicine/Holden Comprehensive Cancer Center core research facility at the University of Iowa. This facility is funded through user fees and the generous financial support of the Carver College of Medicine, Holden Comprehensive Cancer Center, and Iowa City Veteran's Administration Medical Center. Research reported in 
this publication was supported by the National Center for Research Resources of the NIH under award number 1 S10 OD016199-01A1.

Address correspondence to: Massimo Attanasio, University of Iowa, CBRB, 3270B, 285 Newton Road, Iowa City, Iowa 52242, USA. Phone: 1.319.335.9403; Email: massimo-attanasio@uiowa.edu.

1. Coca SG, Singanamala S, Parikh CR. Chronic kidney disease after acute kidney injury: a systematic review and meta-analysis. Kidney Int. 2012;81(5):442-448.

2. Chawla LS, Amdur RL, Amodeo S, Kimmel PL, Palant CE. The severity of acute kidney injury predicts progression to chronic kidney disease. Kidney Int. 2011;79(12):1361-1369.

3. Wang HE, Muntner P, Chertow GM, Warnock DG. Acute kidney injury and mortality in hospitalized patients. Am J Nephrol. 2012;35(4):349-355

4. Liu Y. Cellular and molecular mechanisms of renal fibrosis. Nat Rev Nephrol. 2011;7(12):684-696.

5. Kramann R, et al. Perivascular Gli1 ${ }^{+}$progenitors are key contributors to injury-induced organ fibrosis. Cell Stem Cell. 2015;16(1):51-66.

6. Kramann R, et al. Pharmacological GLI2 inhibition prevents myofibroblast cell-cycle progression and reduces kidney fibrosis. J Clin Invest. 2015;125(8):2935-2951.

7. Yang L, Besschetnova TY, Brooks CR, Shah JV, Bonventre JV. Epithelial cell cycle arrest in G2/M mediates kidney fibrosis after injury. Nat Med. 2010;16(5):535-543.

8. Leaf IA, Duffield JS. What can target kidney fibrosis? Nephrol Dial Transplant. 2017;32(supp1_1):i89-i97.

9. Liu BC, Tang TT, Lv LL, Lan HY. Renal tubule injury: a driving force toward chronic kidney disease. Kidney Int. 2018;93(3):568-579.

10. Kuilman T, Michaloglou C, Mooi WJ, Peeper DS. The essence of senescence. Genes Dev. 2010;24(22):2463-2479.

11. Campisi J, d'Adda di Fagagna F. Cellular senescence: when bad things happen to good cells. Nat Rev Mol Cell Biol. 2007;8(9):729-740.

12. Campisi J. Aging, cellular senescence, and cancer. Annu Rev Physiol. 2013;75:685-705.

13. van Deursen JM. The role of senescent cells in ageing. Nature. 2014;509(7501):439-446.

14. Acosta JC, et al. A complex secretory program orchestrated by the inflammasome controls paracrine senescence. Nat Cell Biol. 2013;15(8):978-990.

15. Tchkonia T, Zhu Y, van Deursen J, Campisi J, Kirkland JL. Cellular senescence and the senescent secretory phenotype: therapeutic opportunities. J Clin Invest. 2013;123(3):966-972.

16. Chien $\mathrm{Y}$, et al. Control of the senescence-associated secretory phenotype by NF- $\mathrm{kB}$ promotes senescence and enhances chemosensitivity. Genes Dev. 2011;25(20):2125-2136.

17. Campisi J. Cellular senescence: putting the paradoxes in perspective. Curr Opin Genet Dev. 2011;21(1):107-112

18. Herranz N, Gil J. Mechanisms and functions of cellular senescence. J Clin Invest. 2018;128(4):1238-1246.

19. Braun H, et al. Cellular senescence limits regenerative capacity and allograft survival. J Am Soc Nephrol. 2012;23(9):1467-1473

20. Westhoff JH, et al. Hypertension induces somatic cellular senescence in rats and humans by induction of cell cycle inhibitor p16INK4a. Hypertension. 2008;52(1):123-129.

21. Liu J, et al. Accelerated senescence of renal tubular epithelial cells is associated with disease progression of patients with immunoglobulin A (IgA) nephropathy. Transl Res. 2012;159(6):454-463.

22. Sturmlechner I, Durik M, Sieben CJ, Baker DJ, van Deursen JM. Cellular senescence in renal ageing and disease. Nat Rev Nephrol. 2017;13(2):77-89.

23. Coppé JP, Desprez PY, Krtolica A, Campisi J. The senescence-associated secretory phenotype: the dark side of tumor suppression. Annu Rev Pathol. 2010;5:99-118.

24. Demaria M, et al. An essential role for senescent cells in optimal wound healing through secretion of PDGF-AA. Dev Cell. 2014;31(6):722-733.

25. Ray P, De A, Min JJ, Tsien RY, Gambhir SS. Imaging tri-fusion multimodality reporter gene expression in living subjects. Cancer Res. 2004;64(4):1323-1330.

26. Laberge RM, et al. Mitochondrial DNA damage induces apoptosis in senescent cells. Cell Death Dis. 2013;4:e727.

27. Fink M, Henry M, Tange JD. Experimental folic acid nephropathy. Pathology. 1987;19(2):143-149.

28. Gupta A, Puri V, Sharma R, Puri S. Folic acid induces acute renal failure (ARF) by enhancing renal prooxidant state. Exp Toxicol Pathol. 2012;64(3):225-232.

29. Stallons LJ, Whitaker RM, Schnellmann RG. Suppressed mitochondrial biogenesis in folic acid-induced acute kidney injury and early fibrosis. Toxicol Lett. 2014;224(3):326-332.

30. Orjalo AV, Bhaumik D, Gengler BK, Scott GK, Campisi J. Cell surface-bound IL-1alpha is an upstream regulator of the senescence-associated IL-6/IL-8 cytokine network. Proc Natl Acad Sci USA. 2009;106(40):17031-17036.

31. Sanz AB, et al. NF-kappaB in renal inflammation. J Am Soc Nephrol. 2010;21(8):1254-1262.

32. Ve T, Gay NJ, Mansell A, Kobe B, Kellie S. Adaptors in toll-like receptor signaling and their potential as therapeutic targets. Curr Drug Targets. 2012;13(11):1360-1374.

33. Sanz AB, et al. NF-kappaB in renal inflammation. J Am Soc Nephrol. 2010;21(8):1254-1262.

34. Leaf IA, et al. Pericyte MyD88 and IRAK4 control inflammatory and fibrotic responses to tissue injury. J Clin Invest. 2017;127(1):321-334.

35. Lemos DR, et al. Interleukin-1 $\beta$ activates a MYC-dependent metabolic switch in kidney stromal cells necessary for progressive tubulointerstitial fibrosis. J Am Soc Nephrol. 2018;29(6):1690-1705.

36. Kim BS, et al. Ischemia-reperfusion injury activates innate immunity in rat kidneys. Transplantation. 2005;79(10):1370-1377. 
37. Mudaliar H, Pollock C, Komala MG, Chadban S, Wu H, Panchapakesan U. The role of Toll-like receptor proteins (TLR) 2 and 4 in mediating inflammation in proximal tubules. Am J Physiol Renal Physiol. 2013;305(2):F143-F154.

38. Igarashi P, et al. Ksp-cadherin gene promoter. II. Kidney-specific activity in transgenic mice. Am J Physiol. 1999;277(4):F599-F610.

39. Shao X, Johnson JE, Richardson JA, Hiesberger T, Igarashi P. A minimal Ksp-cadherin promoter linked to a green fluorescent protein reporter gene exhibits tissue-specific expression in the developing kidney and genitourinary tract. J Am Soc Nephrol. 2002;13(7):1824-1836.

40. Thomson RB, et al. Isolation and cDNA cloning of Ksp-cadherin, a novel kidney-specific member of the cadherin multigene family. J Biol Chem. 1995;270(29):17594-17601.

41. Whyte DA, et al. Ksp-cadherin gene promoter. I. Characterization and renal epithelial cell-specific activity. Am J Physiol. 1999;277(4):F587-F598.

42. Briscoe J, Thérond PP. The mechanisms of Hedgehog signalling and its roles in development and disease. Nat Rev Mol Cell Biol. 2013;14(7):416-429.

43. Li Y, Zhang H, Choi SC, Litingtung Y, Chiang C. Sonic hedgehog signaling regulates Gli3 processing, mesenchymal proliferation, and differentiation during mouse lung organogenesis. Dev Biol. 2004;270(1):214-231.

44. Yu J, Carroll TJ, McMahon AP. Sonic hedgehog regulates proliferation and differentiation of mesenchymal cells in the mouse metanephric kidney. Development. 2002;129(22):5301-5312.

45. Li B, et al. Increased hedgehog signaling in postnatal kidney results in aberrant activation of nephron developmental programs. Hum Mol Genet. 2011;20(21):4155-4166.

46. Shao X, Somlo S, Igarashi P. Epithelial-specific Cre/lox recombination in the developing kidney and genitourinary tract. $J A m$ Soc Nephrol. 2002;13(7):1837-1846.

47. Baar MP, et al. Targeted apoptosis of senescent cells restores tissue homeostasis in response to chemotoxicity and aging. Cell. 2017;169(1):132-147.e16.

48. Gluba A, Banach M, Hannam S, Mikhailidis DP, Sakowicz A, Rysz J. The role of Toll-like receptors in renal diseases. Nat Rev Nephrol. 2010;6(4):224-235.

49. Leemans JC, et al. Renal-associated TLR2 mediates ischemia/reperfusion injury in the kidney. J Clin Invest. 2005;115(10):2894-2903.

50. Ma J, et al. TLR4 activation promotes podocyte injury and interstitial fibrosis in diabetic nephropathy. PLoS ONE. 2014;9(5):e97985

51. Baker DJ, et al. Naturally occurring p16(Ink4a)-positive cells shorten healthy lifespan. Nature. 2016;530(7589):184-189.

52. Farr JN, et al. Targeting cellular senescence prevents age-related bone loss in mice. Nat Med. 2017;23(9):1072-1079.

53. Serrano M. Senescence helps regeneration. Dev Cell. 2014;31(6):671-672.

54. Ritschka B, et al. The senescence-associated secretory phenotype induces cellular plasticity and tissue regeneration. Genes Dev. 2017;31(2):172-183

55. Hou B, Reizis B, DeFranco AL. Toll-like receptors activate innate and adaptive immunity by using dendritic cell-intrinsic and -extrinsic mechanisms. Immunity. 2008;29(2):272-282.

56. Lin F, et al. Kidney-specific inactivation of the KIF3A subunit of kinesin-II inhibits renal ciliogenesis and produces polycystic kidney disease. Proc Natl Acad Sci USA. 2003;100(9):5286-5291.

57. Patel V, et al. Acute kidney injury and aberrant planar cell polarity induce cyst formation in mice lacking renal cilia. Hum $\mathrm{Mol}$ Genet. 2008;17(11):1578-1590.

58. Igarashi P, et al. Ksp-cadherin gene promoter. II. Kidney-specific activity in transgenic mice. Am J Physiol. 1999;277(4):F599-F610.

59. Lu D, et al. Loss of Glis2/NPHP7 causes kidney epithelial cell senescence and suppresses cyst growth in the Kif3a mouse model of cystic kidney disease. Kidney Int. 2016;89(6):1307-1323.

60. Hur E, et al. The effects of vitamin D on gentamicin-induced acute kidney injury in experimental rat model. Int J Endocrinol. 2013;2013:313528. 